\title{
Historia
}

\section{La cosmovisión, conjunto articulado de sistemas ideológicos para el conocimiento y comprensión de los cuidados tradicionales}

\section{The cosmovisión, articulated set of ideological systems for the knowledge and understanding of the traditional care} Cosmovisão, grupo articulado de sistemas ideológicos para o conhecimento e entendendo dos cuidados tradicionais

${ }^{*}$ )Artigo oriundo da dissertação de mestrado intitulada Movimento de configuração do espaço social das enfermeiras no campo da saúde em Teresina de 1959 a 1973. Teresina, Piauí, Brasil.

Javier Badillo Zúñiga ${ }^{1}$, Alfredo Bermúdez González ${ }^{2}$

${ }^{1}$ Maestro en Enfermería "Educación en Enfermería” 10a Generación. PME. Becario CONACYT. Medalla Alfonso Caso. Enfermero Especialista C en el Instituto Nacional de Rehabilitación.

${ }^{2}$ Director Asociado de la Asociación Histórico Filosófica del Cuidado y la Enfermería, (AHFICEN AC.) Vicepresidente de la FIAHE. Profesor de Asignatura A definitivo de la Facultad de Estudios Superiores Zaragoza, UNAM.

Cómo citar este artículo en edición digital: Badillo-Zúñiga, J., \& Bermúdez-González, A. (2017). La cosmovisión, conjunto articulado de sistemas ideológicos para el conocimiento y comprensión de los cuidados tradicionales. Cultura de los Cuidados (Edición digital), 21(49). Recuperado de http://dx.doi.org/10.14198/cuid.2017.49.07

Correspondencia: C/ Callejón de las Rosas No. 7. Pueblo de la Candelaria. Coyoacán. C.P. 04380. México.

Correo electrónico: javier_7badillo@yahoo.com.mx

Recibido: 11/03/2017; Aceptado: 15/05/2017

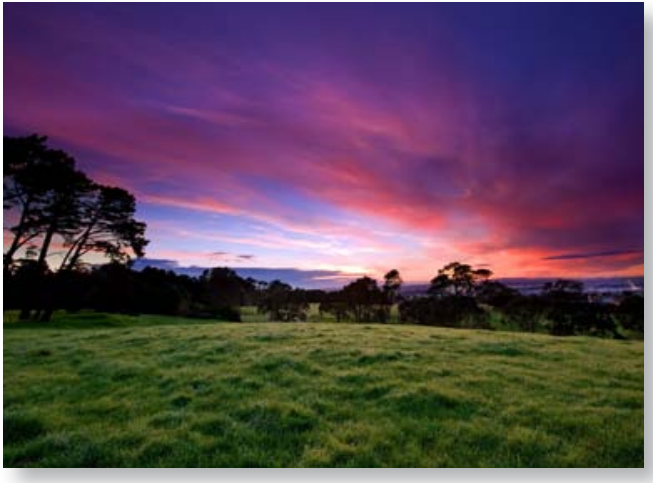

ABSTRACT

A historical and interdisciplinary approach provides specific information on traditional practices such as health care and illness, a historical fact that currently prevails contributing to nursing discipline, which belongs to its object of study. Interpreting the cosmovision as an articulated set of ideological systems for the knowledge and understanding of traditional care, under the historical method and hermeneutical methodology, interpretative paradigm based on the collection of historical, philological, philosophical, ethnographic data, among others, ratifies the interpretation And understanding of the underlying knowledge of the cosmovision of traditional practices such as health care and disease in pre-Hispanic and present indigenous societies. The concept posed by Wilhelm Dhilthey and contextualized for Mesoamerican studies by Johanna Broda and López Austin, is thought to be important for identifying, knowing and understanding traditional practices. The concept of cosmovi- 
sion for the nursing discipline is useful if it is studied as a core of products that identify an articulated set of ideological systems such as traditional practices, their ideological field, origin, characteristics, social dynamics, conception of man, Disease and of course, its care in its humanist dimension.

Keywords: Cosmovision, nursing, traditional practices, health care and illness.

\section{RESUMEN}

Un enfoque histórico e interdisciplinario aporta información específica sobre las prácticas tradicionales como cuidado de la salud y la enfermedad, hecho histórico que prevalece en la actualidad contribuyendo a la disciplina de enfermería, lo que de su objeto de estudio le pertenece. Interpretar a la cosmovisión como conjunto articulado de sistemas ideológicos para el conocimiento y comprensión de los cuidados tradicionales, bajo el método histórico y metodología hermenéutica, paradigma interpretativo basado en la reunión de datos históricos, filológicos, filosóficos, etnográficos, entre otros, ratifica la interpretación y comprensión del conocimiento subyacente de la cosmovisión de las prácticas tradicionales como cuidado de la salud y la enfermedad en las sociedades indígenas prehispánicas y presentes. El concepto planteado por Wilhelm Dhilthey y contextualizado para estudios mesoamericanos por Johanna Broda y López Austin, se piensa trascendente para identificar, conocer y comprender las prácticas tradicionales. El concepto cosmovisión para la disciplina de enfermería es util si se estudia como núcleo de productos que identifiquen un conjunto articulado de sistemas ideológicos como las prácticas tradicionales, su campo ideológico, el origen, características, dinámica social, concepción del hombre, noción de salud, enfermedad y por supuesto, su cuidado en su dimensión humanista.

Palabras clave: Cosmovisión, enfermería, prácticas tradicionales, cuidado de la salud y la enfermedad.

\section{RESUMO}

A abordagem histórica e interdisciplinar fornece informações específicas sobre as práticas tradicionais, tais como cuidados de saúde e doença fato histórico atualmente contribuindo para a disciplina de enfermagem, que de seu assunto pertence prevalecentes. Interpretar a visão do mundo como conjunto articulado de sistemas ideológicos para conhecimento e compreensão dos cuidados tradicionais sob o método e hermenêutica metodologia histórica, paradigma interpretativo baseado na reunião do histórico, filológico, filosófico, etnográfico, entre outras coisas, confirma a interpretação e compreender o entendimento subjacente da visão de mundo de práticas tradicionais, tais como cuidados de saúde e doença nas sociedades indígenas pré-hispânicos e presentes. O conceito proposto por Wilhelm Dhilthey e contextualizada para estudos mesoamericanas por Johanna Broda e López Austin é pensado importante identificar, conhecer e compreender as práticas tradicionais. A cosmovisão conceito para a disciplina de enfermagem é útil se você estudar como o núcleo de produtos que permita identificar um conjunto articulado de sistemas ideológicos como práticas tradicionais, seu campo ideológico, a origem, características, dinâmicas sociais, concepção do homem, conceito de saúde, doença e, claro, o seu cuidado na sua dimensão humanista.

Palavras-chave: Visão de mundo, cosmovisão, enfermagem, práticas tradicionais, cuidados de saúde e doença. 


\section{INTRODUCCIÓN:}

El presente artículo se desprende de la tesis de maestría en educación en enfermería, impartida por la unidad de posgrado de la Universidad Nacional Autónoma de México. La tesis fue realizada bajo una metodología poco convencional para la disciplina de enfermería. El paradigma interpretativo y el método histórico desde donde se obtuvieron diferentes interpretaciones y analogías que sustetaron epistémicamente las prácticas tradicionales para el cuidado de la persona de un pueblo original como los nahuas de la época prehispánica y su presencia en la actualidad. Es desde donde cabe sustraer y resaltar la aplicación del concepto cosmovisión en un estudio de enfermería, tomando en cuenta la importancia del conocimiento que la cosmovisión posee, inclusive, como eje de búsqueda para el conocimiento y comprensión de las prácticas tradicionales con las que cuidan la salud y la enfermedad los diversos y múltiples sociedades indígenas y no indígenas del territiorio nacional.

Se entiende a la cosmovisión como engranaje de ideas y creencias, desde donde se originan acciones específicas y dirigidas bajo una lógica propia que está sujeta a una forma de ver el mundo, al hombre, a la salud, la enfermedad y la muerte, de las cuales subyacen las formas de cuidado de la persona, su particularidad y diversidad, por lo que se considera apropiado un enfoque interdisciplinario. Planteamientos que anclan lo que se debe entender por cosmovisión es lo planteado por el doctor Carlos Viesca (1986), quien menciona que la cosmovisión a sido un concepto desde donde es posible el acercamiento a culturas representativas de desarrollos complejos como las de Mesoamérica prehispánica, totalmente distinto a los patrones y pensamiento europeo.
Debe recordarse que resaltar la importancia del concepto cosmovisión, tiene su formulación en el pensamiento del filósofo de nacionalidad alemana Wilhelm Dilthey; resalta Fernández (2009) en el término Weltanschauung, que se traduce como concepción del mundo o visión del mundo, el cual permitió identificar e interpretar diversos fenómenos que se vinculan estrechamente a partir de las explicaciones con las que dotan a la naturaleza, su relación y vínculo, al tiempo que dan forma a los conceptos e ideas concebidas por un grupo de personas.

En la precisión de situar la investigación, la definición de cosmovisión está enfocada a identificar, conocer, clasificar, interpretar $y$ comprender las visión del mundo de los pueblos mesoamericanos, por lo que es posible visibilizar su influencia en las prácticas tradicionales para el cuidado de la persona en un pueblo originario, los nahuas de la época prehispánica, a partir del acercamiento historiográfico, desde donde es posible realizar la interpretación de nociones conceptuales de su naturaleza, es decir, los significados de las prácticas tradicionales, su naturaleza, la interacción real y no real con la que explican la naturaleza de su creación y existencia, la idea del hombre en la vida, lo cual proporciona una noción de lo que integra al hombre en el conjunto articulado de ideas sobre sí mismo, permitiendo ver la ideología de diversas características que se configuran en los grupos que conforman una sociedad, determinando desde sus propias perspectivas, las interpretaciones a los fenómenos de la vida colectiva y cotidiana, como lo son aquellas prácticas tradicionales enfocadas en el cuidado de la salud y la enfermedad de una persona.

Por lo tanto, resaltar la importancia del concepto cosmovisión y su aplicabilidad en el 
campo de la enfermería desde un estudio de tipo histórico, hace recordar lo que menciona el investigador López (1994), ...la visión del mundo es más que una herramienta del etnólogo..., así también debe ser entendido, como se ha mencionado, desde la aplicabilidad de lo que aporta el trabajo interdisciplinario a la disciplina de enfermería.

La cosmovisión es para la presente investigación, la importancia de conocer y comprender la visión del mundo en una persona, familia o comunidad a la que se pretende otorgar cuidados para la salud, sobre todo en comunidades indígenas, áreas rurales, religiones e ideologías, donde se hace preciso conocer y comprender los cuidados desde su visión del mundo, las prácticas tradicionales que realizan en su vida cotidiana para cuidar su salud y las enfermedades o inclusive, la usencia de éstas; es decir, ante la diversidad de las prácticas tradicionales para el cuidado de la salud y la enfermedad como fenómeno cultural, se plantea la necesidad de que en todo estudio que sean situaciones humanas, como lo es el cuidado de la salud acción que dará permanencia en la vida al hombre, se deben establecer los criterios con respecto a la estructuración del universo, a las articulaciones entre sus partes, el papel y valor del hombre dentro del esquema que encierra su cosmovisión, esto debe ser realizado y posteriormente considerado como una acción de cuidar bajo dimensiones ética, profesional, investigativa y humanista.

Resaltando el sentido de la palabra alemana Weltanschauung utilizada por Broda y Baez (2001) para ubicar el concepto en estudios de los pueblos indígenas mesoamericanos, manifiesta que la cosmovisión es:

...la visión estructurada en la cual los antiguos mesoamericanos combinaban de manera coherente sus nociones sobre el medio ambiente en que vivían, y sobre el cosmos en que situaban la vida del hombre. Así como El estudio de la cosmovisión plantea explorar las multiples dimensiones de cómo se percibe culturalmente la naturaleza. El término alude a una parte del ambito religioso y se liga a las creencias, a las explicaciones del mundo y al lugar del hombre en relación con el universo, pero de ninguna manera puede sustituir el concepto mas amplio de la religión...

Conocer los cuidados tradicionales de las personas debe entenderse como parte del conocimiento del ser humano, lo cual compete a la disciplina de enfermería, ya que la comprensión de la persona es para otorgar cuidados profesionales, personalizados, conociendo necesidades culturales, ideológicas como parte de la integralidad que otorga el conocimiento de su cosmovisión.

Finalmente se concluye que la cosmovisión es un concepto clave dentro de un enfoque histórico e interdisciplinario para enfermería, bajo la orientación de concebir diferentes aportaciones a un gran número de eslavones que vinculan prácticas tradicionales para el cuidado de la salud y la enfermedad, mezcladas con creencias, rituales, sujetos a roles sociales específicos, que se encuentra influenciados por una cosmovisión milenaria que persiste en la actualidad.

\section{METODOLOGIA}

El método histórico a enfermería otorga un sentido básico para la comprensión de la realidad centrada en los saberes, conocimiento históricamente vinculado a su origen histórico, centrado en el paradigma de la enfermería para realizar la búsqueda e interpretación, sujetándose a la mirada de una epistemología analógica que ayudará a la comprensión del propio paradigma, ya que proceder la inter- 


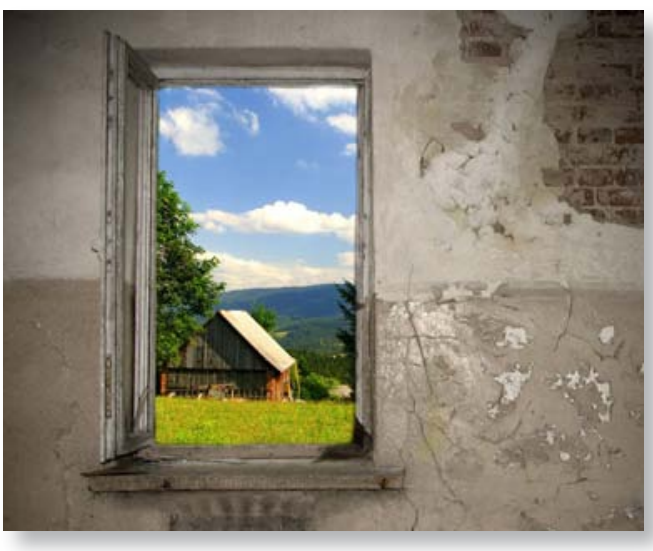

pretación desde paradigmas permitirá mostrar lo que queremos, al tiempo que hacernos comprender en la teoría y actuar en la práctica, manifiesta Khun (2007) y posteriormente resalta Beuchot (2009). La Localización de las fuentes se realizó bajo la búsqueda de autores especialistas en el tema, las palabras clave, referencias bibliográficas en acervos de las bibliotecas, "Samuel Ramos" de la Facultad de Filosofía y Letras, UNAM; Biblioteca "Rubén Bonifaz Nuño" del Instituto de Investigaciones Filológicas, UNAM; Biblioteca "Graciela Arroyo de Cordero" de la Escuela Nacional de Enfermería y Obstetricia, UNAM; Biblioteca "Rafael García Granados" del Instituto de Investigaciones Históricas de la UNAM; Biblioteca Nacional de México a resguardo de la UNAM; bases de datos y revistas electrónicas como Estudios de Cultura Náhuatl. Se realizó la revisión y clasificación de la información, como parte de un ejercicio heurístico con la identificación de hallazgos. Finalmente se empleó la metodología Hermenéutica, al respecto del interés por abordar la tradición como fuente de conocimiento, menciona Gadamer (1993) que: El comienzo de toda hermenéutica histórica debe hallarse por lo tanto en la resolución de la oposición abstracta entre tradición e investigación histórica, entre historia y conocimiento de la misma., argumentando que la tradición que se mantiene y el efecto de la investigación histórica forman una unidad cuyo análisis permite encontrar un entramado o red de semejanzas reciprocas, que se deben reconocer dentro del contexto de la tradición en su comportamiento histórico y elucidar su propia productividad hermenéutica, interpretativa.

\section{DESARROLLO}

Se parte de la premisa que el concepto cosmovisión es conocer, interpretar y comprender. Se reconoce a la cosmovisión como un engranaje de ideas y creencias, donde se originan acciones específicas y dirigidas bajo una lógica propia que está sujeta a una forma de ver el mundo, al hombre, a la salud, la enfermedad y la muerte, de las cuales se piensa subyacen las formas de cuidado de la persona, su particularidad y diversidad, en este sentido las disicplinas aportan sus resultados sobre un fenómeno, que ya sea por vinculación o acción de semejanza, proporcionan información relevante que ensancha el marco de referencias, por ello se piensa apropiado el enfoque interdisciplinario en una investigación de enfermería.

El presente estudio del cual se desea resaltar la importancia del concepto cosmovisión, formulado por primera vez por el filósofo de nacionalidad alemana Dilthey (1948) en el término Weltanschauung, que se traduce como concepción del mundo o visión del mundo ${ }^{2}$, permite identificar e interpretar diversos fenómenos que se vinculan estrechamente con el cuidado de la salud en los nahuas prehispánicos, al tiempo que otorga conceptos e ideas concebidas desde el horizonte de la cosmovisión, dejando ver que dicho concepto encierra una concepción específica para conocer y comprender a un grupo humano, a su vez que visibiliza lo que han desarrollado como conocimiento. 
En el propio sentido de la ubicación de los grupos humanos a estudiar, su tiempo y territorio geográfico, es que la doctora Broda (1991) aporta una forma de entender la cosmovisión de los mesoamericanos, donde menciona que la cosmovisión es una ...visión estructurada en la cual los antiguos mesoamericanos combinan de manera coherente sus nociones sobre el medio ambiente en que vivían, y sobre el cosmos en que situaban la vida del hombre. De tal forma que las nociones sobre el medio en que vivían, permean cualquier práctica o ritual que realizaban para cuidar la salud, la vida.

Uno de los estudiosos que ha enfocado sus análisis en la cosmovisión que tenian los pueblos originarios y su relación con la visión del hombre es el doctor López (2008), quien define cosmovisión como: ...el conjunto articulado de sistemas ideológicos relacionados entre sí en forma relativamente congruente, con el que un individuo o un grupo social, en un momento histórico, pretende aprehender el universo, el cual, sólo surge de las relaciones sociales. Por lo que la cosmovisión debe ser identificada como la base de las características de la dinámica que rige todas las cosas, las actividades, la ideología de una persona, familia, comunidad o colectivo, donde lo “...relativamente congruente...", queda en el discurso, ya que como realidad vivida en las personas que han corporeizado tal realidad, no puede ser relativa y sí, congruente con su verdad y debe ser conocida y comprendida, sobre todo en aquellos procesos de existencia o ausencia del cuidado de la salud y la enfermedad.

De tal manera que conocer la cosmovisión que impera en los pueblos precolombinos, esta directamente relacionada a la búsqueda de la referencia histórica de un paradigma, el cuidado de la persona; es decir, es necesario contemplar todos los fenómenos implicados en las conductas sociales, colectivas, en gremio e individuales que se desarrollen alrededor de un fenómeno específico como hecho histórico. A propósito, el doctor Portilla (2006 expone una necesidad metodológica bajo la cual construye su obra, Filosofía Náhuatl. Estudiada en sus fuentes., tal punto metodológico es expuesto refiriéndose al antropólogo Manuel Gamio con la nota que cita: ...porque no se puede calificar en ningún sentido aquello de que no se tiene conocimiento, y que por primera vez se contempla, no puede ser apreciado ni estimado suficientemente para calificarlo... Por otro lado y sobre la importancia de abordar la cosmovisión, refiere el doctor Portilla (2006) que para comprender la vida indígena,

...es pues necesario empaparse de la mentalidad indígena, conocer sus antecedentes, sus mitos, su cosmografía, su filosofía, en una palabra hay que adquirir los moldes genéricos del pensamiento indígena; para ello, hay que comprender a fondo, integralmente, cualquier aspecto o manifestación de la cultura, es menester reconstruir humanísticamente todos los aspectos de su cosmovisión y de ser posible de lo más elaborado de ésta, su filosofía.

La cosmovisión es para la presente investigación, la fuente de conocimientos y respuesta a la necesidad de saber sobre: un sistema ideológico que explique la constitución y el desarrollo de los procesos vitales, considerado así, al cuidado de la salud y la enfermedad, así como las formas que va dando a través de los significados, los referentes históricos y parte sustancial de una ideología que permitirá conocer las manifestaciones de todos los ámbitos ligados al cuidado. Al respecto y como referente de afirmación para el presente trabajo, menciona el doctor López (2008) que: 
La cosmovisión puede equipararse en muchos sentidos a la gramática, obra de todos y de nadie, producto de la razón pero no de la conciencia, coherente y con un núcleo unitario...

...está presente en todas las actividades de la vida social, y principalmente en aquellas que comprenden los distintos tipos de producción, la vida familiar, el cuidado del cuerpo, las relaciones comunales y las relaciones de autoridad.

Considerar a la cosmovisión como un ente que ejerce poder sobre la interpretación de las cosas y la conducta de las personas, que incluye el fenómeno del cuidado y las características con lo que pudiese dotarlo; se piensa que cuando éste está otorgado desde esta visión cosmogónica que le dio nota a la interacción social, a la creación de roles y por lo tanto de especialistas para atender el proceso salud-enfermedad, apoyados en el enunciado de López (2008), se afirma que ... las concepciones del organismo humano guiaron y justificaron el comportamiento práctico de los distintos componentes de la sociedad... en los pueblos nahuas, acuñados, explicados y siempre sujetos a la cosmovisión.

Al respecto de reconocer a las prácticas tradicionales como prácticas para el cuidado de la salud debe entenderse el concepto "práctica" puede ser utilizado como sustantivo o adjetivo, ejemplo del primero es "la práctica de enfermería"; del segundo, leer en voz alta es práctico. De cualquier modo en ambos existe la noción de algo que se realiza, la acción, y que requiere determinado conocimiento para que los resultados sean los esperados. Cuando el término es usado como sustantivo, se está hablando de cualquier oficio, actividad o acción que se realice de manera constante y con compromiso para que no sea un evento parti- cular y sirva para designar un área de servicios específicos.

En el caso de las prácticas tradicionales, tienen que ver con un conjunto de actividades sujetas a patrones culturales, que se han ido concibiendo en el devenir histórico, por lo que contienen un sustento ideológico de valores, creencias, costumbres, mitos y leyes, son transmitidas de generación en generación, ya sea por tradición oral u otra pedagogía.

$\mathrm{Al}$ respecto de la defensa de la tradición según Guenon (1945), otorga el valor de encontrarse en el orden de lo supra-humano, en este sentido corresponde a situaciones y actividades que se encuentran configuradas bajo la vía simbólica, en muchas ocasiones con atributos de lo sagrado, entre estas actividades, las prácticas tradicionales para el cuidado de la salud y la enfermedad.

Por lo tanto las prácticas tradicionales se refieren al sustantivo de la palabra práctica para determinar un oficio o actividad, que aunado al término tradicional, conjuntan la definición de actividades de sustento ideológico que se vinculan con símbolos, ideas de fe y procedencia de tipo sagrado.

En este contexto ideológico, se encuentra lo que a partir de la cosmovisión se ha ido construyendo, organizando, replanteando, muchas veces reflejado en el lenguaje, valores morales, éticos, espirituales, conocimiento de la naturaleza, del hombre y su sentido en la vida.

Es importante resaltar que en el lenguaje popular la conjunción de los términos "prácticas tradicionales", son utilizados en diferentes ámbitos, por ejemplo prácticas tradicionales comerciales, como el "trueque; en la música y la danza, donde se interpretan historias; pero en el caso de las prácticas tradicionales para la salud y enfermedad se sujetan a la definición de tradición que coloca al término en el orden 
de lo supra-humano como plantea Guénon (1945), ya que existen múltiples ideas determinadas por la cosmovisión que interpretan y simbolizan de forma muy particular los fenómenos de la naturaleza, creando con ello complejos ideológicos que van configurando la noción sobre el cuidado de la salud de la persona.

En la salud y las prácticas tradicionales para mantener y recuperar la salud existe un clima místico que Aguirre (1992) interpreta de la siguiente manera: ... los atributos sagrados de los sacerdotes, la ira incontrolable de los dioses es causa de enfermedad y en el diagnóstico y en el tratamiento de las dolencias intervienen fuerzas divinas.

Centrado en un especialista del tema, el doctor López (1994) determina que dentro de "particulares formas de acción", se consideran en el presente trabajo como prácticas tradicionales para el cuidado de la salud, un sistema ideológico como conjunto articulado de elementos ideológicos (representaciones, ideas y creencias), delimitados por particulares formas de acción, con las que un individuo o un grupo social opera sobre un ámbito particular del universo; tal sistema ideológico que permea el ámbito de la salud, la enfermedad, el dolor y la muerte del organismo humano, actúan con el fin de restituir la salud y prolongar la vida, esto es interpretado como el cuidado de la vida.

Todas las prácticas tradicionales enfocadas a la salud que hasta la actualidad persisten desde la época prehispánica, tales como el temazcalli (baño de vapor), herbolaria, masoterapia, limpias, partería, e inclusive el uso de la palabra, se encuentran inmersas en una cosmovisión donde la ritualidad es justificada como parte del cuidado. Este sentido de ritualidad otorga una interacción especial entre el cuidador y la persona cuidada, ya sea mujer, hombre, en diferentes etapas de la vida, ya sea niño, recién nacido, adulto mayor, adolescente, ya que al realizar las prácticas tradicionales se identifica un valioso valor con sello humanista, otorgado a partir de las manifestaciones sobre el conocimiento profundo del ser humano, su origen sagrado y la dignidad humana que tal cosmovisión implica.

\section{CONCLUSIONES}

- El concepto cosmovisión se plantea desde un enfoque interdisciplinario e histórico para ensanchar el conocimiento y afinar la definición del objeto de estudio de la enfermería, el cuidado de la persona, de tal forma que permita crear un vínculo mas humano entre su teoría y la práctica con la que se interacciona con la persona.

- Si bien la cosmovisión es un determinante para la interpretación de las cosas de la vida, entonces el cuidado también está influenciado por la cosmovisión, así como todas las formas de conocimiento popular, de tal forma que para conocer los cuidados y sus prácticas tradicionales en una localidad, grupo social, étnico, ideológico o religioso, es preciso y se deberá investigar y conocer la cosmovisión desde donde fueron erigidos.

- La cosmovisión para enfermería es util si se visualiza y usa como base de productos de conjunto articulado de sistemas donde se observen las relaciones de las prácticas tradicionales y cotidianas, las cuales se van erigiendo desde una particular forma de percibir el mundo, siempre condicionada por una ideologia o tradición que marca el camino del actuar humano en su sociedad y la forma de interpretar la naturaleza de las cosas de la vida y su cuidado. 
- Para enfermería la cosmovisión es una mirada hacia aquello que de la cultura subyace como conocimiento propio del cuidado de la persona, de tal forma que las prácticas tradicionales deben ser revitalizadas como una tradición capaz de reconstituirse a partir de su rescate y la capacidad de renovación.

\section{REFERENCIAS BIBLIOGRÁFICAS}

- Aguirre, B. G. (1992) Medicina y Magia, El proceso de aculturación en la estructura colonial, México: Universidad Veracruzana, Instituto Nacional Indigenista, Gobierno del Estado de Veracruz, Fondo de Cultura Económica.

- Beuchot M. (2009). Tratado de hermenéutica analógica. Hacia un nuevo modelo de interpretación. México: UNAM. ITACA.

- Broda J. y Baez-Jorge F. (2001). Cosmovisión, ritual e identidad de los pueblos indígenas de México, Introducción, México: Fondo de Cultura Económico.

- Broda, J. (1991).Cosmovisión y observación de la naturaleza: el ejemplo del culto de los cerros., en Broda J. Iwaniszewski, S. Maupome, L. Arqueoastronomía y et- noastronomía en Mesoamérica. México: Instituto de investigaciones Históricas. UNAM.

- Dilthey, W. (1948). Introducción a las ciencias del espíritu. México: Fondo de Cultura Económica. Recuperado de https://filosinsentido.files.wordpress. com/2013/05/135001148-dilthey-wilhelm-introduccion-a-las-ciencias-del-espiritu-1883.pdf

- Fernández, L. F. (2009). Wilhelm Dilthey. Enciclopedia Filosófica on Line.

- Gadamer - H. G. (1993). Verdad y Método. Madrid (España): Ediciones Sígueme.

- Guenon, R. (2001), Tradición y tradicionalismo en capítulo XXXI de El Reino de la Cantidad y los signos de los Tiempos. Símbolos, Revista de Arte - Cultura - Gnosis.

- Kuhn, T. S. (2007). La estructura de las revoluciones científicas. México: Fondo de Cultura Económica.

- López A. A. (1994) Tamoanchan y Tlalocan. México: Fondo de Cultura Económica.

- López. A. A. (2008). Cuerpo humano e ideología. Las concepciones de los antiguos nahuas. México: Instituto de Investigaciones Antropológicas, UNAM.

- Portilla L. M. (2006) La Filosofía Náhuatl. Estudiada en sus fuentes, México: UNAM.

- Viesca T. C. (1986). Posibilidades para abordar el estudio de la Medicina Náhuatl, México: Estudios de Cultura Nahuatl.

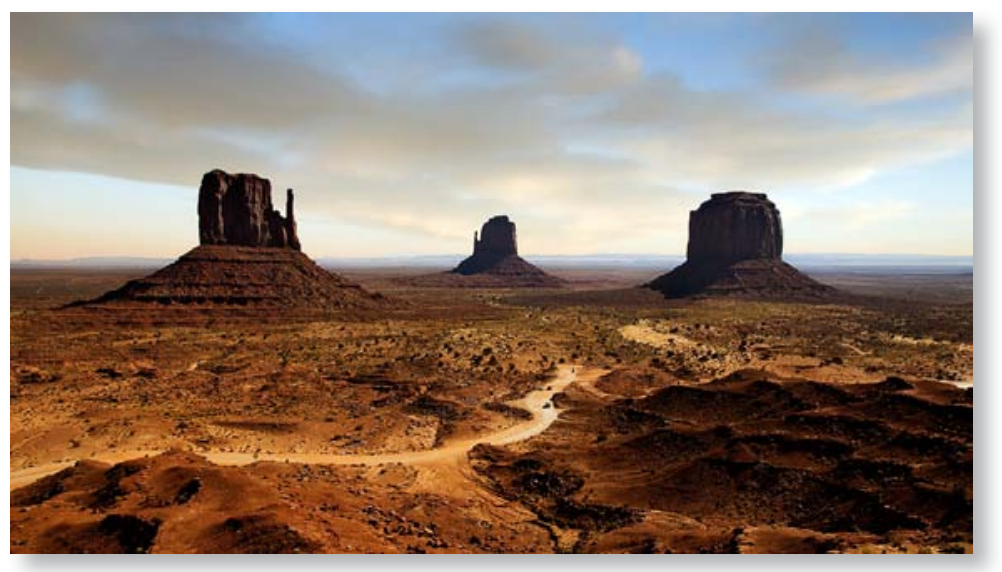

